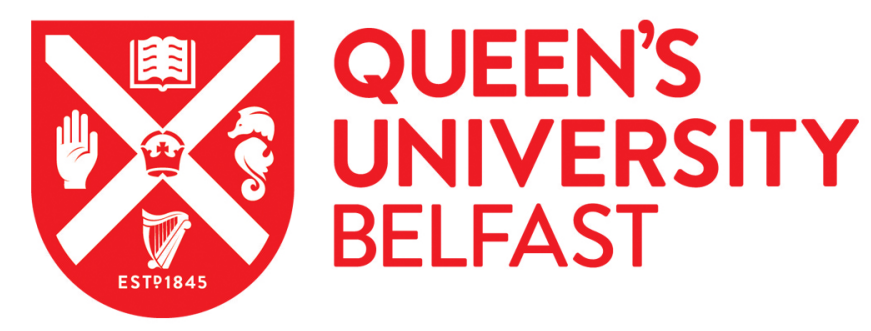

\title{
Localizationlike effect in two-dimensional alternate quantum walks with periodic coin operations
}

Di Franco, C., \& Paternostro, M. (2015). Localizationlike effect in two-dimensional alternate quantum walks with periodic coin operations. Physical Review A (Atomic, Molecular, and Optical Physics), 91(1), [012328]. https://doi.org/10.1103/PhysRevA.91.012328

Published in:

Physical Review A (Atomic, Molecular, and Optical Physics)

Document Version:

Peer reviewed version

Queen's University Belfast - Research Portal:

Link to publication record in Queen's University Belfast Research Portal

Publisher rights

@2015 American Physical Society

\section{General rights}

Copyright for the publications made accessible via the Queen's University Belfast Research Portal is retained by the author(s) and / or other copyright owners and it is a condition of accessing these publications that users recognise and abide by the legal requirements associated with these rights.

Take down policy

The Research Portal is Queen's institutional repository that provides access to Queen's research output. Every effort has been made to ensure that content in the Research Portal does not infringe any person's rights, or applicable UK laws. If you discover content in the Research Portal that you believe breaches copyright or violates any law, please contact openaccess@qub.ac.uk. 


\title{
Localization in two-dimensional alternate quantum walks with periodic coin operations
}

\author{
Carlo Di Franco and Mauro Paternostro \\ Centre for Theoretical Atomic, Molecular and Optical Physics, \\ School of Mathematics and Physics, Queen's University, Belfast, BT7 1NN, United Kingdom
}

\begin{abstract}
Exploiting multi-dimensional quantum walks as feasible platforms for quantum computation and quantum simulation is attracting constantly growing attention from a broad experimental physics community. We propose a modification of the quantum walk scheme described in [C. Di Franco et al., Phys. Rev. Lett. 106, 080502 (2011)] that presents, in the considered regimes, a strong localization-like effect on the walker. We characterize it in terms of the parameters of a step-dependent qubit operation that acts on the coin space before any standard coin operation, showing that a proper choice can guarantee a non-negligible probability of finding the walker at the origin even for large times. We finally discuss possible experimental realizations of this model with the current state-of-the-art settings.
\end{abstract}

The field of quantum computation and quantum simulation has been recently driven to a new rising edge by the experimental realization of quantum walks in various setups, highlighting that different physical systems can be adapted for the implementation of these models. In particular, optical systems have shown their full potential, allowing the experimental demonstration of two-dimensional quantum walks for the first time [1,2], even if several further progresses will surely be obtained also in the other physical scenarios that have been already exploited for the one-dimensional case [3, 4].

From the theoretical point of view, the interest in twodimensional quantum walks has been boosted by the fact that, differently from the one-dimensional version, higherdimensional schemes (i.e., walkers moving on structures with dimension larger than one) can be exploited for the efficient implementation of quantum search algorithms [5]. In particular, the Grover walk has been intensively studied due to its localization feature [6]. It has been proved that the nonlocalized case of the Grover walk can be simulated by a walk where the requirement of a higher dimensionality of the coin space is substituted with the alternance of the directions in which the walker can move [7].

In the quest for more feasible quantum walk models, an important step forward is presented in this paper: a strong localization-like effect can be indeed obtained in the modified version of the alternate quantum walk presented here, reducing the gap with the standard two-dimensional version. This result could pave the way for adapting this scheme to the realization of quantum algorithms, providing a clear advantage in terms of experimental resources. After a short introduction on the quantum walk studied here, we characterize it in terms of its relevant parameters and show that a proper choice can guarantee a non-negligible probability of finding the walker at the origin even for large times. We then hint at a qualitative relation between the ability of the system to localize and the behavior of coherences established in the state of the particles performing a two-particle equivalent scheme of the walk, which in turns provides information on the way correlations are set up between them. We discuss the robustness of this model to imperfections and its possible experimental realization with the current state-of-the-art settings in linear optics and cold-atom devices.
Let us consider a quantum system with two degrees of freedom, and thus described by a vector in the composite Hilbert space $\mathcal{H}=\mathcal{H}_{W} \otimes \mathcal{H}_{C}$. The coin space $\mathcal{H}_{C}$ is a two-dimensional Hilbert space spanned by $\{|0\rangle,|1\rangle\}$ and the walker space $\mathcal{H}_{W}$ is an infinite-dimensional Hilbert space spanned by $\{|x, y\rangle\}$, with $x$ and $y$ assuming all possible integer values. We take as a basis of this space $\mathcal{H}$ the set $\{|x, y, c\rangle\}$, with $|x, y, c\rangle=|x, y\rangle_{W} \otimes|c\rangle_{C} ; x$ and $y$ could denote, for instance, the position of a particle (walker) along the $x$ and $y$ directions, respectively, while $|c\rangle_{C}$ is an internal twolevel degree of freedom. From now on, we consider $|0,0\rangle_{W}$ as the initial state of our walker, and $\left|{ }_{y}\right\rangle_{C}=\left(|0\rangle_{C}+i|1\rangle_{C}\right) / \sqrt{2}$ as the coin one.

The evolution of the system is given by a sequence of conditional shift, coin operations, and phase gates. The effect of the two different conditional shift operations

$$
\hat{S}_{x}=\sum_{i, j \in \mathbb{Z}}|i-1, j, 0\rangle\left\langle i, j, 0\left|+\sum_{i, j \in \mathbb{Z}}\right| i+1, j, 1\right\rangle\langle i, j, 1|
$$

and

$$
\hat{S}_{y}=\sum_{i, j \in \mathbb{Z}}|i, j-1,0\rangle\left\langle i, j, 0\left|+\sum_{i, j \in \mathbb{Z}}\right| i, j+1,1\right\rangle\langle i, j, 1|
$$

is to move the walker on a two-dimensional plane, in a way that depends on the coin state. If we label the position in the $x$ and $y$ directions with increasing numbers from left to right and from bottom to top, respectively, $\hat{S}_{x}$ moves the walker one step to the left (right) when the coin-component is in the state $|0\rangle_{C}\left(|1\rangle_{C}\right)$ and $\hat{S}_{y}$ moves the walker one step down (up) when the coin-component is in the state $|0\rangle_{C}\left(|1\rangle_{C}\right)$. Our coin operation (acting only on the coin space) is the Hadamard gate

$$
\hat{H}=\frac{1}{\sqrt{2}}\left(\begin{array}{cc}
1 & 1 \\
1 & -1
\end{array}\right),
$$

as in the original quantum walk [8]. In order to include some dependence on the (discrete) time (similarly to what has been done in Ref. [9]) in the single walk step, we also add the phase gate

$$
\hat{P}_{\phi}(t)=\left(\begin{array}{cc}
e^{-i \frac{\phi}{2} t} & 0 \\
0 & e^{i \frac{\phi}{2} t}
\end{array}\right)
$$




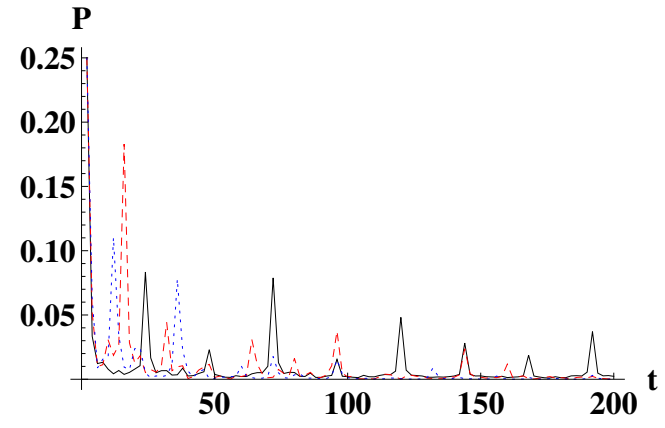

FIG. 1: Probability to find the walker in the origin, against the total number of time steps $t$, for values of $\phi$ equal to $\pi / 6$ (black line), $\pi / 4$ (red dashed line), $\pi / 3$ (blue dotted line). Only the odd time steps are shown, as the probability is zero for all even steps.

with $t$ the corresponding time step of the quantum walk [also $\hat{P}_{\phi}(t)$ acts only on the coin space]. A single time step consists here of two Hadamard operations, two phase gates and two movements on the $x$ and $y$ directions, according to the sequence: coin operation - phase gate - movement on $x$ - coin operation - phase gate - movement on $y$.

Let us first consider the case where both the phase gates (the one before the movement on $x$ and the one before the movement on $y$ ) have the same $\phi$. We are interested in the spatial probability distribution after a fixed number of steps, that can be obtained by tracing out the state of the coin. In particular, we want to investigate the probability to find the walker in the origin, and check if it quickly decreases with time, or any localization-like effect is present. Fig. 1 shows this probability for different values of $\phi$ (we have investigated several other random values of $\phi$, finding always a similar behavior). For comparison, let us remember that in the case $\phi=0$ (i.e., the standard alternate quantum walk) this probability is a nonincreasing function of the time. However, for $\phi \neq 0$, there are peaks showing a return of the walker in the origin.

It would be interesting to check the walker behavior for long times. In order to do that, we consider the return probability (i.e., the probability to be in the origin after a certain number of steps $t$ ), averaged over $t$ (or, more precisely, over $t / 2$, as the probability is zero for all even steps). We plot it against $t$ in Fig. 2, for the same values of $\phi$ as in Fig. 1. We can notice that the average probability rapidly goes to zero. Also in this case, we have investigated several random values of $\phi$, finding always a similar behavior.

Due to the freedom given by the fact that the scheme in Refs. [7] separates the movement of $x$ and $y$, another possibility is clearly to have different phase gates before the two movements, i.e. having different $\phi$. Let us define $\phi_{x}\left(\phi_{y}\right)$ the angle in the phase gate before the movement on $x$ (movement on $y$ ). We can therefore investigate the whole region of the $\phi_{x}-\phi_{y}$ plane and check for localization-like effects. In order to do that, as a reasonable trade-off between the computational power required by the simulation and the readability of the plot, we fix the total number of steps to $t=40$ and

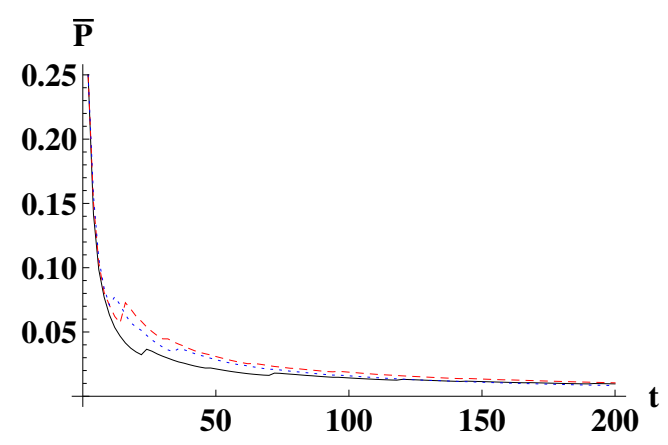

FIG. 2: Average probability to find the walker in the origin, against the total number of time steps $t$, for values of $\phi$ equal to $\pi / 6$ (black line), $\pi / 4$ (red dashed line), $\pi / 3$ (blue dotted line).

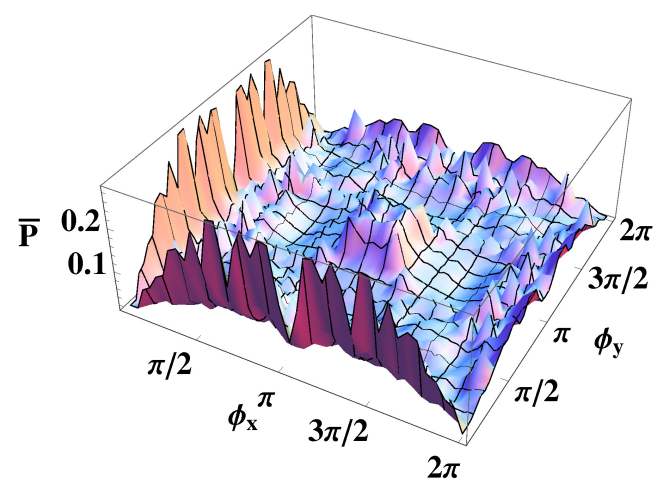

FIG. 3: Average probability to find the walker in the origin, for a total number of time steps $t=40$, against the values of $\phi_{x}$ and $\phi_{y}$.

study the average return probability against the two angles, as shown in Fig. 3. It is straightforward to notice in this plot (as well as in other plots that we obtained for different values of the total number of steps $t$ ) that the maximal values are reached when only one of the two $\phi$ 's is different from zero. Moreover, the average probability is symmetric with respect to $\phi_{x} \leftrightarrow 2 \pi-\phi_{x}, \phi_{y} \leftrightarrow 2 \pi-\phi_{y}$, and $\phi_{x} \leftrightarrow \phi_{y}$.

If we want to find the proper choice of $\phi$ parameters in order to maximize the localization-like effect, and then study its long-time behavior, we can thus just fix one of the $\phi$ 's equal to zero (in the following investigation, we choose $\phi_{y}=0$ ), and vary the other. This will allow us to reduce the number of parameters and investigate longer times. We plot the obtained results in Fig. 4, where also more points have been taken for the values of $\phi_{x}$. The total number of step is $t=100$, in this case. We can notice that there are wide regions where the average return probability is much larger than the value corresponding to $\phi_{x}=\phi_{y}=0$ (i.e., the stardard alternate walk, for which the value is around 0.025). For comparison, for the same number of time steps, when $\phi_{x}=\phi_{y} \neq 0$ (the case analyzed previously), the average return probability never goes above 0.1 . We have already a strong evidence that this choice $\left(\phi_{x} \neq 0, \phi_{y}=0\right)$ could guarantee a localization-like effect.

We thus take the value for which we have the maximum at $t=100\left(\phi_{x}=19 / 25\right)$ and we plot again the average return 
probability against $t$ (as done in Fig. 2) for this case. The difference is evident: The probability does not go rapidly to zero and seems to have an asymptotic value. It is interesting to notice that a localization-like effect has been observed in one-dimensional quantum walk with periodic coin operation as well [9], but in that case it is a transition behavior before the walker starts to spread. A localization effect could strikingly widen the possible applications of the alternate quantum walk, considering in particular the importance of localization in the Grover walk [5], the two-dimensional quantum walk exploited for the implementation of Grover search algorithm.

If the model has to be used for realistic implementation, it is clearly necessary to analyze how robust it is against imperfections. A systematic error in the value of $\phi_{x}$ will not strongly affect the localization-like effect, as it can be already noticed in Fig. 4. Even if the value of $\phi_{x}$ is not exactly the one corresponding to the maximum, wide regions of $\phi_{x}$ give reasonable return probability. The other possibility is that the error on $\phi_{x}$ is not systematic. For instance, at each time step the angle could assume a value equal to $(1+\delta) \phi_{0}$, where $\phi_{0}$ is the ideal value and $\delta$ is a random variable taking into account possible inaccuracies. In order to check the robustness, we have thus studied the return probability sampling $\delta$ at each time step from a uniform distribution in the range $[-0.01,0.01]$ (corresponding to a possible error up to $1 \%$ of the optimal value). We present the results, averaged over 100 trails, in Fig. 6.

There are two regions that can be distinguished in the plot. The first one is for short times, where the effect of the periodicity in the operation acting on the coin space keeps the return probability quite high, very close to the optimal value in Fig. 5. Then, in the second region, the probability starts to decay, due to the effect of randomization of the the phase angle. The value is still higher than the standard alternate quantum walk, but the probability clearly goes to zero for long times. This can be understood by considering that disorder in the quantum walk coin operation can have some localization effect as well [10]. However, three different kinds of disorder can appear: one only depending on the time (i.e., a fixed value of $\delta$ at each time step, as we have considered previously), one only on the position (i.e., a fixed value of $\delta$ at each position of

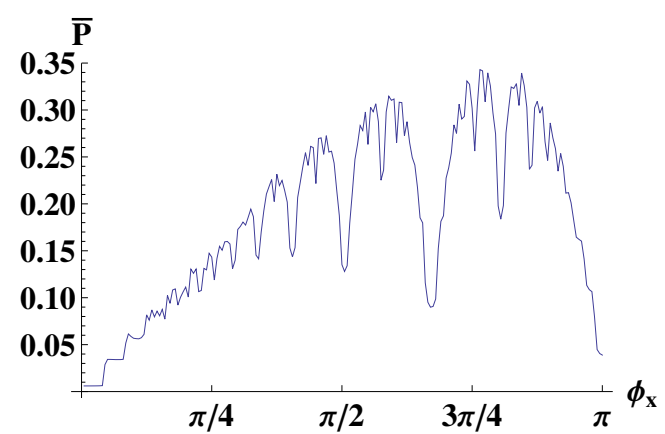

FIG. 4: Average probability to find the walker in the origin, for a total number of time steps $t=100$ and $\phi_{y}=0$, against the values of $\phi_{x}$.

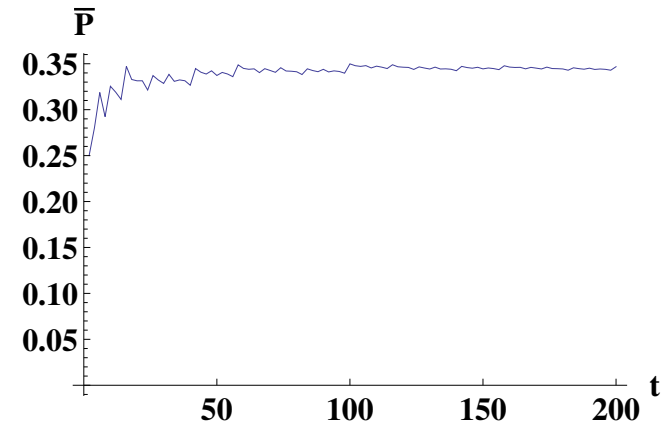

FIG. 5: Average probability to find the walker in the origin, against the total number of time steps $t$, for $\phi_{x}=19 / 25$ and $\phi_{y}=0$.

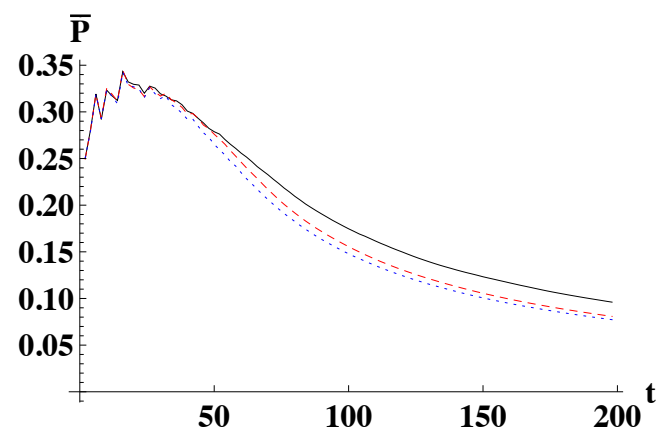

FIG. 6: Average probability to find the walker in the origin, against the total number of time steps $t$, for a value of $\phi_{x}=(1+\delta) \phi_{0}$, $\phi_{0}=19 / 25, \delta$ sampled at each time step from a uniform distribution in the range $\left[-10^{-2}, 10^{-2}\right]$, and $\phi_{y}=0$. The black line corresponds to time-dependent disorder, the red dashed line corresponds to position-dependent disorder, the blue dotted line corresponds to disorder depending both on position and time.

the lattice on which the walker is moving), and one on both. In particular, only the second gives rise to Anderson localization effects, even if the other two still have localization effects. In all the three cases, however, the probability to find the walker in the origin goes to zero for the time going to infinity. For the sake of completeness, we have studied the other two cases and reported also these results in Fig. 6 (red dashed and blue dotted lines, respectively).

It is very interesting to compare the localization phenomenon to the behavior of coherences established between the particles performing a two-particle equivalent scheme of the walk. As described in Ref. [11], the alternate quantum walk can also been seen from a different point of view: two particles both moving on a line, not interacting directly but sharing a common degree of freedom, embodied by the coin. The one-dimensional movement of the first particle corresponds to the shift of the original walker on the $x$ direction, and the one-dimensional movement of the second particle to the shift of the original walker on the $y$ direction. Intuitively, one expects that the achievement of localization in the alternate walk would affect the way the two walkers are able to correlate each other, establishing some form of localization- 


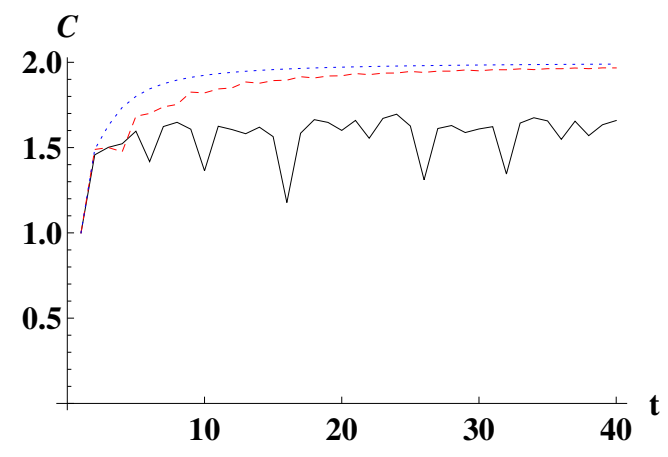

FIG. 7: Coherence norm against the total number of time steps $t$, for $\phi_{x}=19 / 25, \phi_{y}=0$ (black line), $\phi_{x}=\pi / 4, \phi_{y}=0$ (red dashed line), and $\phi_{x}=0, \phi_{y}=0$ (blue dotted line).

radius. Only within such radius the motion along the $x$ direction would be tied to that along the $y$ direction. In turn, this would be reflected in the way off-diagonal elements in the two-walker density matrix are populated as the number of steps in our process grows. In the non-localized case, on the other hand, we expect sizeable coherences to be established even when the walkers are beyond the localization-radius described above.

In order to provide a quantitative assessment of such expectations, we have studied the behavior of the quantity

$$
\mathcal{C}=\left\|\rho_{W}-\rho_{W, x} \otimes \rho_{W, y}\right\|
$$

with $\|A\|=\operatorname{Tr} \sqrt{A^{\dagger} A}$ the trace-norm of an operator $A, \rho_{W}$ the density matrix of the walkers and $\rho_{W, k}(k=x, y)$ its reduction for the walker align the $k$ axis. We $\operatorname{dub} \mathcal{C}$ as the coherence norm. By providing the sum of the moduli of the off-diagonal elements of the density matrix $\rho_{W}$, the coherence norm is adopted, in our study, as a quantifier of the global behavior of walker-walker coherences. Notice that a very recent investigation has put forward similar tools as genuine measures of coherence in a quantum system [12]. We have compared the trend followed by the coherence norm for three different arrangements of the walk, namely one case of strong localization $\left(\phi_{x}=19 / 25, \phi_{y}=0\right.$, black line), one case of weak localization $\left(\phi_{x}=\pi / 4, \phi_{y}=0\right.$, red dashed line), and one case of no-localization $\left(\phi_{x}=0, \phi_{y}=0\right.$, blue dotted line). The results, presented in Fig. 7, show that $\mathcal{C}$ displays rather distinctive features and confirm our predictions. As coherence in the extended Hilbert space of the two walkers is directly related to the correlations established by the two particles moving along the orthogonal axes of our lattice, this suggests in a quantitative and measurable way a strong connection between the phenomenology of inter-walker correlations and the features of the walk itself, an aspect that clearly deserves further in-depth investigation.

Let us now discuss possible experimental implementations of this model. The standard alternate quantum walk has been recently realized in a optical loop setup [2]. In order to obtain the model proposed here, we should add a phase gate after the first coin operation (denoted as "coin 1" in Figure 1 of Ref. [2]). This can be implemented by means of an electrooptic modulator (EOM), as done in Ref. [13] for demonstrating the effects of disorder in one-dimensional case. As shown in Ref. [13], the EOM can be properly programmed in order to change the phases between single steps of the quantum walk, therefore allowing the dependency of the phase gate operation on the time step, as required in the proposed model. Clearly, this time-dependent alternate quantum walk can also be realized in any other physical setups suitable for the standard alternate version. For instance, when an alternate quantum walk will be implemented by means of neutral atoms in optical lattices, along the lines of the one-dimensional experiment in Ref. [3], the only change to obtain the model studied here will be in the operation acting on the internal degree of freedom (1.e., in the pulse allowing transitions between the considered hyperfine levels of the atoms).

We have studied a time-dependent alternate quantum walk obtaining, in the considered regimes, a strong localizationlike effect on the walker. We have investigated this behavior and found the optimal strategy to enhance it. This could pave the way for adapting the scheme to the realization of feasible quantum algorithms, providing a clear advantage in terms of experimental resources. We have also shown that it can be experimentally implemented with the current state-of-the-art technology.

Acknowledgments.- MP is grateful to S. F. Huelga for invaluable discussions on the issue of coherence in quantum processes. The authors acknowledge financial support from the UK EPSRC under the Career Acceleration Fellowship and "New Directions for EPSRC Research Leaders" schemes (EP/G004579/1), and the John Templeton Foundation (grant ID 43467).

[1] A. Schreiber et al., Science 336, 55 (2012).

[2] Y-C. Jeong et al., Nat. Commun. 4, 2471 (2013).

[3] M. Karski et al., Science 325, 174 (2009).

[4] H. Schmitz et al., Phys. Rev. Lett. 103, 090504 (2009); F. Zähringer et al., Phys. Rev. Lett. 104, 100503 (2010); M. A. Broome et al., Phys. Rev. Lett. 104, 153602 (2010).

[5] N. Shenvi, J. Kempe, and K. B. Whaley, Phys. Rev. A 67, 052307 (2003); A. Ambainis, J. Kempe, and A. Rivosh, in Proc. 16th ACM-SIAM SODA, Vancouver (SIAM, Philadelphia, USA, 2005), p. 1099; A. Tulsi, Phys. Rev. A 78, 012310 (2008).

[6] N. Inui, Y. Konishi, and N. Konno, Phys. Rev. A 69, 052323 (2004); M. Štefaňák, T. Kiss, and I. Jex, Phys. Rev. A 78, 032306 (2008).

[7] C. Di Franco, M. Mc Gettrick, and Th. Busch, Phys. Rev. Lett. 106, 080502 (2011); C. Di Franco, M. Mc Gettrick, T. Machida, and Th. Busch, Phys. Rev. A 84, 042337 (2011).

[8] Y. Aharonov, L. Davidovich, and N. Zagury, Phys. Rev. A 48, 1687 (1993).

[9] M. C. Bañuls et al., Phys. Rev. A 73, 062304 (2006).

[10] A. Ahlbrecht et al., J. Math. Phys. 52, 042201 (2011); A. Joye, Comm. Math. Phys. 307, 65 (2011); A. Joye and M. Merkli, J. Stat. Phys. 140, 1 (2010); A. Ahlbrecht, V. B. Scholz, and A. H. 
Werner, J. Math. Phys. 52, 102201 (2011); A. Ahlbrecht et al., arXiv:1201.4839 (2013).

[11] C. Di Franco, M. Mc Gettrick, T. Machida, and Th. Busch, J. Comput. Theor. Nanosci. 10, 1613 (2013).
[12] F. Levi, and F. Mintert, arXiv:1310.6962 (2013); T. Baumgratz, M. Cramer, and M. B. Plenio, arXiv:1311.0275 (2013). [13] A. Schreiber et al., Phys. Rev. Lett. 106, 180403 (2011). 